Revista de Psicología de la PUCP. Vol. XIX, 1, 2001

\title{
La experiencia subjetiva de la enfermedad en pacientes con esquizofrenia
}

\author{
Bertha Kaliksztein Fihman ${ }^{1}$ \\ Pontificia Universidad Católica del Perú
}

\begin{abstract}
La presente es una investigación de nivel descriptivo que explora la experiencia de la enfermedad en quince pacientes esquizofrénicos desde su propia perspectiva mediante una entrevista semiestructurada. Los testimonios más significativos fueron la atribución de la enfermedad a la falta de orientación por parte de los padres; la percepción de dificultades en las capacidades de aprendizaje y atención desde la etapa escolar, y las conflictivas y escasas relaciones interpersonales. El tener una ocupación, la ayuda psicológica y el gozar de tranquilidad fueron señalados como aspectos que contribuirían a una mejoría. Sentimientos como el miedo, la depresión, la intranquilidad, entre otros son aspectos inherentes a la vivencia de la enfermedad y creemos que prestarles atención ayuda a comprender mejor la experiencia de la esquizofrenia.

Palabras clave: experiencia subjetiva, esquizofrenia, fenomenología de la enfermedad.
\end{abstract}

\section{Subjective experience of the disease in patients with schizophrenia}

This descriptive investigation explores experimentation of the disease in 15 schizophrenic patients from the perspective of the patients themselves through semistructured interviews. The most significant testimonies were the attribution of the disease to a lack of orientation from the parents, the perception of difficulties in learning and attention abilities in class and the scarce and conflictive interpersonal relationships. Having an occupation, psychological support and stability in their lives were mentioned as aspects that contribute to significant improvement. Feelings like fear, depression, lack of peace among others are inherent aspects of the disease. In our opinion listening to the patient helps to better understand the experiencing of schizophrenia.

Keywords: subjective experience, schizophrenia, phenomenology of the illness.

1. Licenciada en Psicología por la PUCP. Bachiller en Sociología y Antropología en la Universidad de Tel-Aviv, Israel. Jefa de Práctica de la Especialidad de Psicología de la PUCP. 

La esquizofrenia es un tipo especial de psicosis que se caracteriza por múltiples y distintas áreas de funcionamiento perturbadas que configuran una manera irreal de apreciarse a sí mismo y al mundo. Las consecuencias poco favorables de adaptación al medio, la falta de integración de la personalidad, la cronicidad y el deterioro concomitante hacen de esta una de las enfermedades mentales más graves conocidas hasta hoy. Se trata de una entidad clínica que incluye (Atkinson, 1986; American Psychiatric Association, Diagnostic and statistical manual of mental disorders IV, 1994): (a) perturbaciones del pensamiento, (b) perturbaciones de la percepción, (c) perturbaciones de la atención, (d) perturbaciones de la voluntad, (e) perturbaciones motrices, $(\mathrm{f})$ perturbaciones emocionales y $(\mathrm{g})$ perturbaciones en las relaciones interpersonales; esto significa que no todos los pacientes tendrán los mismos síntomas ni experimentarán la enfermedad de la misma manera.

La mayoría de autores ha coincidido en que por lo general existió un turbulento conflicto, tensión, ansiedad, hostilidad o desapego general en la familia del paciente desde sus años formativos. Afirman además que esto no es suficiente para explicar la esquizofrenia, pero sí es una condición necesaria (Arieti, 1974).

Alrededor de los años 50, la esquizofrenia fue foco de atención dentro del círculo psicoanalítico. Fromm-Reichmann (1973) considera al esquizofrénico como una persona que ha tenido graves experiencias traumáticas en la temprana infancia, en una época en la que su yo y su aptitud para examinar la realidad todavía no estaban desarrolladas. Sechehaye (1958) afirma que a medida que la enfermedad evoluciona, se da la pérdida de la síntesis del yo; así, la esquizofrenia es para ella una enfermedad ante todo del yo, pues se presenta una irrupción del inconsciente y esta invasión no puede ocurrir sino a expensas de una desintegración del yo. 
Cada vez existen más y más evidencias de que la esquizofrenia es una severa perturbación del funcionamiento cerebral. Randolph, Goldberg y Weinberger (1993) concluyeron que existen ciertas disfunciones cerebrales inherentes a la esquizofrenia desde antes de la instalación de la sintomatología psicótica, como los déficits de atención, memoria y funciones ejecutivas. Van den Bosch (1995) afirma que la esquizofrenia es un desorden cognitivo-neuropsicológico subjetivamente experimentado puesto que muchas de las anormalidades comportamentales pueden ser entendidas como estrategias adaptativas para afrontar a los déficits cognitivos. Refiere que varios síntomas de la esquizofrenia son el resultado de intentos compensatorios, sea buscando significado o esfuerzos autoprotectores orientados a reducir el estrés mental, o a "comprender" las experiencias extrañas.

Cincuenta años de investigación no han podido producir una prevención efectiva o cura para la esquizofrenia y esto sea quizá porque no se ha descubierto ninguna causa definitiva. Hoy en día, mediante el uso de medicación antipsicótica y psicoterapia, los síntomas positivos de la enfermedad pueden ser controlados. Yung et al. (1998) han demostrado que es posible identificar individuos con una alta probabilidad de instalación de la enfermedad dentro de un breve período de tiempo y afirman que se puede proporcionar un tratamiento que apunté a prevenir, demorar o minimizar la severidad de la instalación de la esquizofrenia.

\section{La experiencia subjetiva de la enfermedad}

Las nociones, actitudes, sentimientos, percepciones y expectativas desarrolladas por los pacientes respecto de su enfermedad constituyen lo que llamamos la experiencia subjetiva de la enfermedad.

Arieti (1974) refiere que el paciente esquizofrénico de alguna manera siente que no vive la plena realidad de la vida "[...] como si algo no natural y extraño lo separara del mundo" (p. 105). Tausk (Roheim, 
La experiencia subjetiva de la enfermedad en pacientes con esquizofrenia

1959) enfatiza que muchos pacientes creen estar bajo la influencia de algo extraño, otros se quejan de sentir una extrañeza interior - el individuo se siente "extraño" ante sí mismo-. Racamier (1983) ha señalado: "de lo que carece fundamentalmente el psicótico es del sentimiento íntimo de una familiaridad esencial entre el yo y la realidad, pierde toda familiaridad con lo real" (p. 105). A los mismos enfermos les cuesta trabajo encontrar la palabra adecuada para expresar su estado de ánimo. Conrad (1964) señala que unos lo describen como presión o tensión, inquietud, angustia, a veces también como animación alegre, como ocurre en la esperanza. Otros, lo experimentan como culpa y condenación, como si tuvieran pendiente un castigo o hubiesen cometido un crimen. Otros, a su vez, no se sienten más que inhibidos, sin gana, sin voluntad, abandonados y sin esperanza. "La depresión es un aspecto común en el primer episodio de la esquizofrenia $[. .$.$] en mucha gente el primer episodio incluso coincide con$ los criterios de un episodio de depresión mayor [...]" (D. Addington, J. Addington y Patten, 1998).

Conrad (1964) señala que el paciente se preocupa por lo que las personas no dicen y lo que, de una manera oculta y a sus espaldas, hacen, intentan, maquinan, hablan entre sí mientras él no está presente; "de esta manera brota el sentimiento de perjuicio y desconfianza" (p. 193). El paciente, señala Arieti (1974), distorsiona el ambiente y experimenta una gran vulnerabilidad, miedo, ansiedad y dolor. Cree que es y será inaceptable por los otros, que no encaja, está solo y se siente muy solo. Esto lo lleva a desorganizarse y a sufrir un inmenso terror.

Arieti (1974) señala que a veces el paciente da poca importancia al fenómeno de las voces, pero otras veces atraviesa una experiencia repentina, profunda y conmovedora. Escucha una voz poderosa o sonido con un mensaje dirigido a él, solo a él, un mensaje que está relacionado con todo su ser psicológico. Luego el paciente espera tener las alucinaciones y efectivamente las tiene. Finalmente, estas últimas se pueden convertir en un fenómeno familiar y su ocurrencia es tan 
frecuente que el paciente las acepta como una parte importante de su vida.

Corin (1990) ha señalado que para entender mejor el mundo esquizofrénico debemos apreciar la enfermedad desde la perspectiva de la experiencia esquizofrénica. De igual manera, Conrad (1964) ha mencionado la importancia de apoyarse en los factores vivenciales para la comprensión de la enfermedad.

\section{Metodología}

El presente es un estudio exploratorio con niveles descriptivos. El tipo de diseño es no-experimental transeccional de medición única.

\section{Participantes}

Trabajamos con quince pacientes diagnosticados con esquizofrenia, hospitalizados en un Instituto Nacional de Salud Mental de Lima, adultos y de ambos sexos. Se procuró seleccionar a quienes presentaban un tiempo de enfermedad similar y un diagnóstico de esquizofrenia estable en el tiempo según su historia clínica.

\section{Instrumentos}

Se utilizó una entrevista semiestructurada en temas elegidos de acuerdo con los objetivos de la investigación. Estos temas fueron los siguientes: la conciencia de enfermedad, las manifestaciones de la enfermedad, los cambios a partir de la enfermedad, cómo afecta la enfermedad, las fantasías en torno a la enfermedad, el significado de la enfermedad y las expectativas que tiene el paciente para el futuro. Además de la entrevista, se utilizó una ficha de datos para cada participante, en ella se consignó información complementaria. Una vez seleccionados los participantes, se procedió con las sesiones de entrevista que fueron grabadas con la previa autorización de cada partici- 
pante y luego transcritas. El análisis de los datos se realizó con la técnica de análisis de contenido.

\section{Resultados}

Los resultados de la investigación han sido organizados en temas seleccionados a partir de la información revisada, así como según el material recogido en las entrevistas a los pacientes. Los temas incluyen categorías establecidas de acuerdo con las respuestas de los pacientes.

\section{Conciencia de enfermedad}

El análisis de esta dimensión de la enfermedad se realizó sobre la base de los cuatro modos de experiencia subjetiva de la esquizofrenia descritas por León (1992) en su estudio. El autor señala que después de un tiempo, variable de paciente a paciente, se consolida una noción subjetiva de la experiencia psicótica y esta constituye un núcleo vivencial alrededor del cual se organiza un sistema de ideas, percepciones, actitudes y sentimientos relativos a la enfermedad y sus consecuencias: lo que llamó el modo de experiencia subjetiva de la enfermedad. Aquí se distingue cuatro categorías: integración, se refiere a la capacidad del paciente de incorporar la experiencia del trastorno dentro de la continuidad de la vida psíquica; el paciente asimila la idea de haber enfermado mentalmente. Atenuación, el paciente tiende a disminuir el impacto emocional causado por el trastorno, calificándolo como un estado de "nerviosidad", "preocupaciones" y "problemas". Negación, el paciente no recuerda o pretende no recordar todo lo relativo al trastorno. Finalmente, falsificación en la cual se dan interpretaciones distorsionadas de la realidad, no existe percepción del trastorno psíquico como tal y el contacto con la realidad está alterado en forma total o parcial. 
En la Figura 1 se puede apreciar cómo los entrevistados se distribuyeron en las categorías mencionadas. Resulta sorprendente que más del $70 \%$ de los pacientes de la muestra tengan una conciencia de enfermedad, por lo menos parcial, dado que en la literatura se habla de porcentajes bastante más bajos. Por conciencia de enfermedad estamos aludiendo a la aceptación y al reconocimiento de un desorden mental, lo cual les permite hablar de él y de las vivencias que este ocasiona, sin embargo, no nos referimos aquí a un entendimiento profundo de la esquizofrenia ni a una capacidad de darse cuenta de las implicancias que tiene el trastorno.

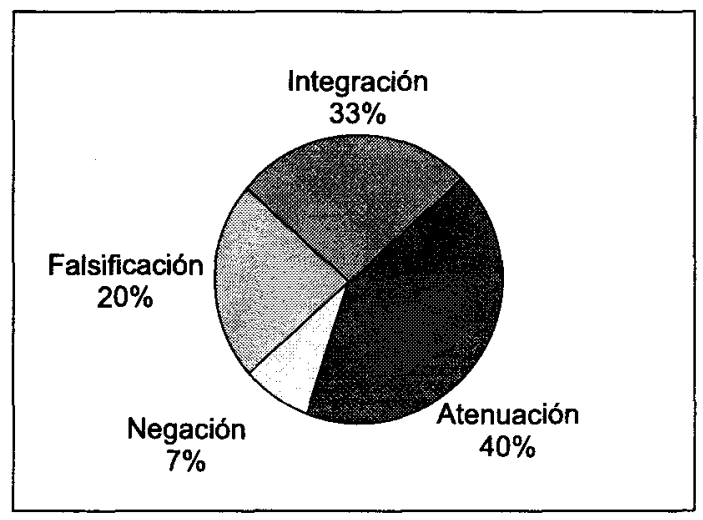

Figura 1. Distribución de los participantes según el modo de experiencia subjetiva.

Algunos pacientes saben que requieren un tratamiento y saben que su enfermedad no tiene una cura definitiva mientras que otros, a pesar de saber que sufren de alteraciones mentales, siguen pensando que quizá trabajando y con la ayuda de Dios podrían combatir las recaídas. Aquellos que tienen un mayor grado de conciencia de enfermedad, los pacientes integrados, son capaces de distinguir -retrospectivamente- la época en que están con la sintomatología propia del trastorno de la época en que se sienten mejor; al mismo tiempo, 
saben que tienen una enfermedad para la cual necesitan medicamentos constante y permanentemente.

\section{El comienzo de la enfermedad}

Los entrevistados mencionaron diversos síntomas, sentimientos y vivencias que se dieron al inicio de su enfermedad. Las manifestaciones más frecuentes fueron las alteraciones fisiológicas, las alucinaciones auditivas, la intranquilidad, las delusiones o ideas extrañas y las dificultades para captar/aprender, seguidas de miedo, soledad, trastornos de pensamiento formal, y en menor proporción fueron mencionados el retraimiento, preocupaciones, alucinaciones visuales, cólera, desconfianza y depresión.

\section{La enfermedad de cada uno}

Se le preguntó a cada paciente cuál creía que era su enfermedad o su problema. Siete pacientes hicieron alusión a una enfermedad mental, expresándose de las siguientes maneras: "estoy mal de la cabeza", "estoy mal del cerebro", "he perdido la razón", "sufro de alteraciones mentales", "tengo confusión del cerebro", "tengo un problema del alma". Se encontró una tendencia a reducir la severidad del trastorno en cinco pacientes que refirieron estar enfermos de "nervios"; encontramos expresiones como "muchas preocupaciones", "enfermo de tristeza, estoy muy solo", "intranquilidad", "angustia", "tenía muchas inseguridades, miedos". Un paciente refirió estar enfermo de "cólera" mientras otra paciente mencionó estar enferma de "celos". Solo un paciente negó su enfermedad: "yo no estoy enfermo, no sé por qué me han traído".

\section{Causas de la enfermedad}

Casi todos los pacientes dieron múltiples respuestas, sin embargo, para facilitar el análisis hemos tomado la respuesta más representativa de cada paciente, es decir, a la que el paciente le dio mayor importancia. Las respuestas se resumen en el Cuadro 1: 


\section{Cuadro 1}

Causas de la enfermedad

\begin{tabular}{|lcc|}
\hline & \multicolumn{2}{c|}{ Entrevistados que lo mencionan } \\
\hline Respuesta & $f$ & $\%$ \\
\hline Problemas familiares & 8 & 53.33 \\
Accidente & 2 & 13.33 \\
Creencias religiosas & 2 & 13.33 \\
Desconfianza & 1 & 6.66 \\
Querer trabajar y no poder & 1 & 6.66 \\
No estoy enfermo & 1 & 6.66 \\
\hline Total & 15 & 100.00 \\
\hline
\end{tabular}

Resulta significativo que más de la mitad de los entrevistados respondieron que enfermaron por problemas familiares. Los problemas familiares fueron diversos y los hemos clasificado bajo las siguientes subcategorías: "discusiones familiares" (cinco pacientes), "desunión familiar" (cuatro pacientes) y "falta de orientación por parte de los padres" (cuatro pacientes). Algunas de las respuestas fueron:

"Había discusiones, problemas entre mis padres, mis hermanos, pero más los padres, me he ido poniendo mal poco a poco"; "Mis padres son desunidos, mi mamá tiene una religión y mi papá otra religión, mi padre es católico"; "Es que los padres, mejor dicho mi papá y mi mamá [...] mi papá falleció ya hace 14 años y no tuve ni un padre ni una madre que estuviera a mi atrás y yo por ejemplo yo me quejaba donde mi mamá, pero mi mamá nunca supo que [...] por no hacer caso a otra gente no hubiera enfermado, ¿no? Si hubiera tenido un padre y una madre que fueran profesionales allí sí no me hubiera pasado esto [...] ahí sí me hubieran ayudado, pero lamentablemente no eran, mi mamá tampoco era [...]"; "Mi padre nunca nos aconsejó, nos reprochó, nunca nos dio consejo de padre [...] mi padre nunca nos ha orientado [...]", "Solo pido que mi padre me aconseje, me oriente [...] yo hubiera sido un hombre diferente, 
positivo [...] si mi padre sería otra gente, si mi madre sería otra madre, sería yo otra gente. Más comunicación con mi madre me faltaba [...] no sé, tal vez desconfianza, desarmonía, tal ıez me hace falta una madre, un padre [...] no sé cómo responderle [...]".

\section{El aspecto religioso}

En el transcurso de las entrevistas, la mayoría de pacientes evocó espontáneamente contenidos religiosos. En algunos casos el tema religioso fue ampliado con preguntas dirigidas a profundizar en las vivencias de los pacientes.

Nueve pacientes $(60 \%)$ refirieron que se comunicaban con Dios, sea porque hablaban con Dios o habían tenido algún otro tipo de c atacto como haberlo visto o haber escuchado que los llamaba. En algunos casos un mismo paciente evocó más de una vivencia de índole religiosa. Por ejemplo, una paciente refirió haber "escuchado un llamado de Dios", mencionó también que "Dios sabía por qué había enfermado", y además comentó "haber visto a Dios en las nubes".

Casi la mitad de los pacientes refirieron que el creer en Dios los ayudaba a sentirse mejor o a sobrellevar su enfermedad, lo expresaron de las siguientes maneras:

"Como yo creo bastante en Dios yo pensaba que Dios se iba a encargar de mi enfermedad, ya no me preocupaba de tomar pastillas [...]"; "Todo to he dejado en manos de Dios, toda la carga lo dejé en manos de Dios"; "Por la fe en Dios [...] Dios solucionará todos los problemas".

Un porcentaje similar (33.3\%) refirió haber pecado y haber sido castigado por Dios. Estos pacientes experimentan mucha culpa y por ello afirman que deben dedicarse a servir a Dios. El comentario de un paciente: 
"Yo gritaba llorando 'soy pecador, soy pecador', ahora Jesucristo me va a castigar [...] yo había hecho cosas malas [...] tal vez debería confesarme o dedicarme a la religión de Dios, me siento culpable [...]".

Dos pacientes mencionaron que los médicos y enfermeras son de gran ayuda porque "son instrumentos de Dios":

\section{Cognición}

Dentro de las alteraciones en las funciones cognitivas encontramos por un lado los trastornos del pensamiento y por otro la disminución de habilidades cognitivas como el aprendizaje y la atención. Los trastornos del pensamiento se dieron tanto en el contenido como en la forma del pensar. Cabe señalar que un mismo paciente podía presentar trastomos en la forma y contenido del pensamiento y además haber experimentado una disminución en las habilidades de aprendizaje, atención o ambas.

La mayoría de los pacientes $(80 \%)$ habló sobre sus trastornos en el contenido del pensamiento o delusiones, algunos pacientes estaban conscientes de que estas eran falsas premisas; sin embargo, otros estuvieron delusivos en la propia entrevista. Los contenidos de las delusiones fueron muy variados, además, un mismo paciente podía presentar distintos tipos de delusión. Las delusiones más frecuentes fueron las místicas, seguidas de las delusiones de daño y referencia.

Los trastornos del pensamiento formal experimentados por los pacientes (26.6\%) fueron la fuga de ideas, el pensamiento perseverante, el pensamiento confuso y el bloqueo del pensamiento, pudiendo un mismo paciente presentar varios de estos. Algunos ejemplos:

"Mi mente mucho hablaba, por sí solo hablaba mi mente, mi cabeza, mi cerebro por sí solo hablaba [...] no es porque yo quería pensar que mi mente hablara, sino que mi mente hablaba sola 
[...] parece que yo mismo me estaba haciendo daño de tanto pensar"; "Tengo las ideas que se me vienen muy rápidamente [...]"; "Tenía una confusión en el cerebro, dudas [...] pensaba mucho, demasiado, pensar mucho causa desequilibrio [...]"; "Como que ya no me daba más las ideas, de tanto pensar no podía encontrar una solución, me confundía [...]"; "Sentía como si todas mis ideas se hubieran quedado ahí nomás", "Me enredo con mis propias ideas, entonces como que me bloqueo [...]"; "Mi mente se pone en cero, no piensa en nada".

En un tercio de los pacientes fue observada una disminución en otras habilidades cognitivas como el aprendizaje y la atención. Estas alteraciones fueron experimentadas como dificultades para captar y procesar información, mantener el ritmo de aprendizaje y agotamiento intelectual, y fueron percibidas mayormente en la etapa escolar. Los pacientes refírieron:

“¿Qué tendré en mi mente que no puedo captar? [...] El colegio era mucho esfuerzo para mí, me dolía la cabeza, me sentía cansado, no me salía y ya lo dejaba [...]"; "No captaba, tenía problemas para estudiar, no aprendía"; "No podía hacer tanto esfuerzo (en el colegio)"; "No tenía ese ritmo [para estudiar]"; "Terminé la secundaria y quería descansar un par de años, o 3 años [...] quería descansar de toda actividad intelectual, de todo estudio [...]"

\section{Relaciones interpersonales}

Las relaciones con las demás personas se han visto afectadas en la mayoría de los pacientes entrevistados. Estos muchas veces mencionan espontáneamente las dificultades que tuvieron para relacionarse con los miembros de sus familias, con las personas extrañas y con sus parejas. En ocasiones hablaron de los sentimientos de soledad que experimentaban en el mundo. En la Figura 2 se aprecian las modalidades de relaciones interpersonales mencionadas por los pacientes. 


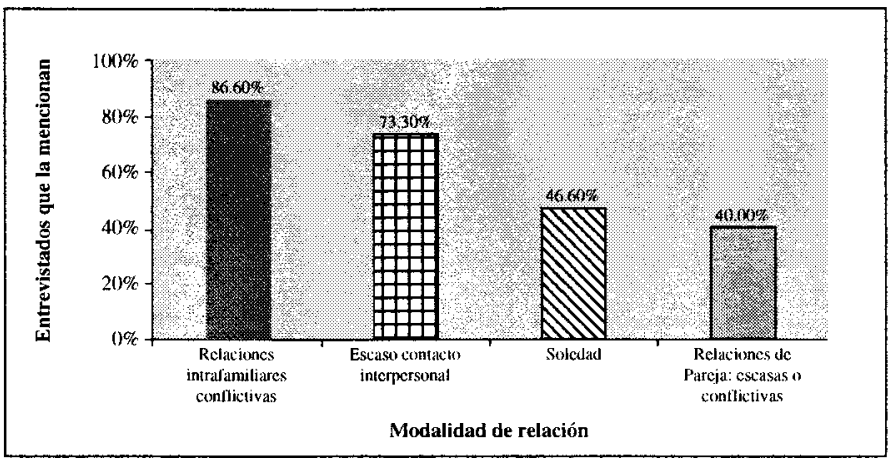

Figura 2. Modalidades de relaciones interpersonales.

Las vivencias de relaciones conflictivas intrafamiliares parecen emerger de la percepción de una falta de orientación por parte de los padres, de la falta de comprensión al interior de sus familias (o ambos) y en algunos casos existe violencia familiar en los hogares de los pacientes. La falta de orientación por parte de los padres - que en algunos casos fue mencionada como causa de la enfermedad fue referida por nueve pacientes. Además de los ya mencionados, los comentarios de algunos pacientes fueron:

"Veo que a veces necesito la orientación de una persona, porque a veces mis padres me quieren orientar pero ellos dentro de sus posibilidades [...]"; "Mi papá me daba propina [...] mi papá no me exigía, mi mamá tampoco, nadie me exigía en mi casa $[\ldots] "$

Más de la mitad de los entrevistados habló acerca de la falta de comprensión entre los miembros de su familia. Algunos pacientes refirieron:

“Mi papá no sé si será ignorante [...] es que mi papá no tiene estudios. Mi papá es ignorante, no me puede entender, no puede entender. Porque él habla, habla, habla nomás, no llega 
a su origen, él habla, grita y hace lo que quiere, dice lo que quiere, entonces yo no le entiendo"; "Necesitaba mucha ayuda, bastante comprensión, porque la comprensión es importante en la familia y en mi casa no la teníamos".

La violencia familiar, verbal y física, tanto por parte de los familiares hacia el paciente como del paciente hacia los familiares:

"Era agresivo con mi familia, primero le di un puñete a mi mamá y después a mi hermano, también le pegué a mis dos hermanos. Le tiré con un cucharón de madera de la cocina, en su cara [...]"; "Sí, también ese comportamiento hostil que tienen mis hermanos que quieren pelear, al ver a la policía agarrar a mis hermanos, eso me produce nerviosismo y me hace a mí dar mucha cólera".

El escaso contacto interpersonal fue mencionado por el $73.3 \%$ de los pacientes. Algunos mencionaron tener pocos amigos, otros contaron sobre su retraimiento y lo poco que socializaron. Algunas razones atribuidas a los escasos contactos fueron: la desconfianza (siete pacientes), miedo a las personas (cinco pacientes) y cuatro pacientes afirmaron que las personas son malas.

Casi la mitad de los pacientes mencionó haber experimentado soledad. Algunos refirieron haber sido personas muy solas desde siempre, otros se han sentido solos en momentos que necesitaban de los demás, mientras que otros hablaron de su incapacidad para establecer relaciones con los demás. Algunos de los comentarios de los pacientes fueron los siguientes:

"Yo más me considero una persona que he estado sola. Me gustaría cambiar un poco. No estar tan sola, sino hablar con mi mamá, con mis hermanos, jugar"; "Yo he sido una persona sola, nunca me ha gustado tener amigos tampoco. Porque 
los amigos a veces se enojan. Solo he estado. No me metía yo con la gente mucho $[\ldots]$ ".

Seis pacientes comentaron que sus relaciones de pareja fueron conflictivas o no las tuvieron, o fueron escasas.

\section{Aspectos que contribuyen a una mejoría}

Luego de escuchar a los pacientes hablar de su enfermedad y sus vivencias en torno a esta, se les preguntó qué les ayudaba a sobrellevar su enfermedad y/o a sentirse mejor. El Cuadro a continuación reúne las respuestas a dichas preguntas.

\section{Cuadro 2}

Aspectos que contribuyen a una mejoria

\begin{tabular}{|lcc|}
\hline & Entrevistados que lo mencionan \\
\hline Respuesta $^{a}$ & $f$ & $\%$ \\
\hline Trabajar & 12 & 80.0 \\
Atención psicológica & 9 & 60.0 \\
Tranquilidad & 8 & 53.3 \\
Medicamentos & 5 & 33.3 \\
Fe en Dios & 5 & 33.3 \\
Comprender la enfermedad & 4 & 26.6 \\
\hline
\end{tabular}

Nota. " Categorías no excluyentes.

Una proporción significativa de los pacientes refirió que trabajar lo ayudaría a sobrellevar su enfermedad o a sentirse mejor. En ocasiones los pacientes expresaron también que el estar ocupados, distraerse o estudiar les haría mucho bien. Las respuestas fueron dadas por pacientes de los cuatro modos de experiencia subjetiva de la enfermedad. Algunos comentarios fueron:

"Trabajando, sirviéndole algo a la vida, servirle a la vida, trabajar, ser algo útil para la sociedad, del país y del mundo [...]"; "Me gusta estar ocupada porque siento que no hacer 
La experiencia subjetiva de la enfermedad en pacientes con esquizofrenia

nada no es bueno, no me hace sentir bien"; "Conseguir un trabajo adecuado que me favorezca y que me ayude bastante"; "Sí, creo que trabajando podría cambiar algo mi vida"; "A veces por ratos digo, no sé si podré volver a la universidad, quiero terminar la carrera de enfermería [...]".

Un número importante de pacientes refirió que un tratamiento psicológico lo ayudaría en su enfermedad. Lo expresaron de diversas maneras, unos comentaron que conversar con un psicólogo los hace sentir bien, otros mencionaron que los psicólogos son los únicos que los comprenden. Algunos comentarios se presentan a continuación:

"Me hace bien aquí dialogar, conversar con usted, que es psicóloga, yo quisiera tener el tratamiento, mejorar mi relación con mi esposa [...] y quizás con el tratamiento psicológico. Defogarme todo lo que tengo adentro, porque así como usted no había hablado con nadie [...]"; "Los profesionales, como usted, los psiquiatras, los psicólogos, ustedes nomás, son los únicos que me ayudan a vivir [...] con ustedes puedo conversar, usted me entiende [...]".

Más de la mitad de los entrevistados afirmó que se sentirían mejor si no hubiera tantos problemas familiares en sus hogares, si tuvieran paz y tranquilidad. Cinco pacientes refirieron que tomar sus medicamentos los ayudaría a estar mejor, algunos incluso mencionaron que se iban a curar tomando sus medicinas. Del mismo modo, cinco pacientes mencionaron que creer en Dios, tener confianza en Dios o dedicarse a la religión los ayuda a sentirse mejor. Finalmente, cuatro pacientes $(26.6 \%)$ manifestaron su interés por comprender su enfermedad o entender algunos aspectos de ella. Lo expresaron de la siguiente manera:

"Yo quiero por eso un diagnóstico completo y real, que yo acepte mi realidad y que yo diga que tiene razón y así comience mi tratamiento. Pero ahorita yo no puedo decir eso 
porque no tengo la certeza [...]"; "Me gustaría tratar de comprender las voces, porque también me dan consejos [...]".

\section{Vivencias esquizofrénicas}

En esta sección del trabajo hemos reunido una selección de citas que describen vivencias profundas y personales de la esquizofrenia. Una paciente describió sus vivencias de despersonalización, habló sobre sus sentimientos de desesperanza y vergüenza y sobre su sufrimiento comparándolo con el infierno:

"Yo me siento muerta en vida, así me siento ahorita. Como si estaría muerta en vida, como así por comer, no tengo ganas de nada, yo misma me aíslo [...] hago un esfuerzo por vivir, no quiero vivir ya, ya le pido a Diosito que me recoja, ya no quiero vivir ya, porque mi enfermedad ya no tiene cura".

"Ya no me tratan como antes. Diferente. Ya no deseo vivir, tengo ya vergüenza de vivir, me da vergüenza".

"Mi enfermedad fue bien peor que un infierno ha sido [...]".

Un paciente describe la esquizofrenia aludiendo a la soledad, al encerramiento y al aislamiento y destaca la falta de comprensión que se tiene respecto de la enfermedad, señalando que solo quien la sufre sabe lo que está atravesando:

"Es una enfermedad que ni los psiquiatras, ni los psicólogos a veces la entienden. Es una enfermedad que uno solo la entiende, cuando uno es inteligente. Hay que enfrentarla, luchar, porque hay psiquiatras que ya no pueden, que sólo con la ayuda de Dios, pero otros profesionales le dan ideas para que nosotros estemos mejor. Porque la esquizofrenia es una enfermedad muy mala, muy mala, es un mundo solo, un mundo aparte de la sociedad. Como si fuera una caverna, te encierra y nunca te saca de ahí. Hasta siglos, hasta siglos. Es bien fea. Yo no puedo describirla más porque sólo Dios puede describirlo, porque él sabe la respuesta a esta enfermedad". 
La experiencia subjetiva de la enfermedad en pacientes con esquizofrenia

El mismo paciente explica la sensación de fragmentación interior que experimenta:

" [...] Como si mi mente es, fuera un equilibrio así [une sus palmas] y ahora se me pasa el tiempo, se mete, se me sale "separa y junta las palmas sin encajar], se mete, se me sale, no puedo unirlo, quiero unirlo para que mi cuerpo sea uno y no puedo, y me da mucho miedo de trabajar, de enfrentar la vida $[\ldots] "$.

Otro paciente usa sus propios términos para resumir y describir las vivencias que le ocasionan la enfermedad, para él se trata de una cuestión de energía. La energía negativa sería todo lo malo de la enfermedad mientras que su opuesto, la energía positiva, es lo que le da fuerzas ante la debilidad:

"Eso es lo que quisiera yo, soltarme un poco más al salir a la calle, al parque, alegrarme un poco más, sentir energía positiva, no negativa. Que también esté parejo para sentirme bien [¿energía negativa?] No sabría explicarle, yo sentía como miedo, retraído, ensimismado [...] el ruido de la basura [triángulo] me hace sentir bien, me da energía, me da poder, me hace sentir como de fierro también, me da fuerza [¿energía negativa?]. Todo lo contrario".

\section{Discusión}

La enfermedad suele comenzar con una serie de manifestaciones que no son siempre las mismas. Un paciente puede reportar un conjunto de síntomas que otro quizá no los tuvo en un inicio. Cuando los pacientes cuentan sobre el inicio de su enfermedad, parecen recordar más las sensaciones corporales y síntomas que están en relación consigo mismos y menos en relación con los otros. Las alteraciones fisiológicas, las voces "al interior de sus cabezas", los trastornos 
del pensamiento, la intranquilidad, y las dificultades para captar información de afuera parecen ocupar más su atención. Cutting y Dunne (1989) han señalado que la mayoría de personas que se han recuperado de una psicosis pueden recordar su primer quiebre psicótico con una gran claridad, incluso muchos años después del evento. "En particular, ellos recuerdan las experiencias anormales en las áreas de percepción, lenguaje, atención, movimiento, pensamiento y sentido espacial" (p. 230). Es importante señalar que fueron precisamente los pacientes integrados - con una mayor conciencia de enfermedadlos que mencionaron vivencias de tipo relacional, como la desarmonía, la desunión, la desconfianza y la envidia.

En varios casos, los pacientes afirmaron que la enfermedad comenzó con alteraciones que podrían resumirse como físico-corporales; nos referimos a las alteraciones fisiológicas como el insomnio y la falta de apetito, y las modificaciones perceptuales-sensoriales como las alucinaciones. Por otro lado, los pacientes señalaron las tempranas dificultades cognitivas en las capacidades de aprendizaje y atención y también dificultades ejecutivas, como la falta de iniciativa, agotamiento, etc. Tanto los déficits atencionales, de memoria y de aprendizaje como los déficits ejecutivos han sido estudiados neuropsicológicamente, encontrando substratos neurológicos responsables para dichos déficits (Randolph et al., 1993). Coincidiendo con este enfoque, en nuestro estudio se observó que en todos los pacientes que experimentaron déficits cognitivos y ejecutivos los síntomas psicóticos fueron posteriores a aquellos, y que algunos déficits cognitivos persistían aún cuando los síntomas psicóticos desaparecían. De este modo, nuestras observaciones reforzarían las hipótesis sobre la existencia de una fuerte base neurobiológica en la esquizofrenia, la cual es responsable no solo de experiencias físico-corporales y sensoriales-perceptuales, sino probablemente también de otras experiencias menos tangibles y mensurables, cognitivas y afectivas, como las delusiones, los trastornos de pensamiento formal y el afecto aplanado. Lo que se desconoce aún es en qué medida se produciría la combinación de 
La experiencia subjetiva de la enfermedad en pacientes con esquizofrenia

estos factores con los otros factores ambientales y cómo influyen los unos sobre los otros.

Casi todos los pacientes comentaron sobre el ambiente familiar poco grato que vivieron en sus hogares desde la infancia. Este hecho refuerza el consenso de la mayoría de autores que estudiaron la esquizofrenia psicodinámicamente, que por lo general existió conflicto, tensión, ansiedad, hostilidad o desapego en la familia del paciente desde sus años formativos (Arieti, 1974). Si bien cada paciente vivió estas dificultades de manera única, muchos de ellos hablan sobre experiencias similares. La falta de orientación por parte de los padres no solo fue señalada como una dificultad que vivieron, sino también como una de las causas de su enfermedad. Esta falta de orientación alude a una falta de dirección, consejo y apoyo con los que esperaban poder contar en sus vidas. Algunos refieren que todo hubiera sido distinto - y quizá no hubieran enfermado - si hubieran tenido alguna figura que cumpla dicho rol. Un paciente concluyó que sus padres no lo prepararon para la vida, mientras que otro mencionó la falta de un padre y una madre, a pesar de tenerlos físicamente.

Todo esto nos hace pensar en que no hubo quién regule desde fuera aquello que los pacientes empezaban a sentir en su interior, como lo es la sensación de desintegración y frente a ella la necesidad de que alguien los ayude a no desmoronarse; como el sentirse incapaces de desenvolverse en un medio social, intelectual y laboral y, por ende, la necesidad de una guía, apoyo o pautas para lograr una mejor adaptación; como la soledad y los temores que experimentaban $\mathrm{y}$ ante estos la necesidad de que alguien se ponga de su lado y los conforte, intente explicar estos sentimientos atemorizantes, se hiciera cargo de llenar vacíos y sustituir un mundo interno no gratificante. $\mathrm{O}$ por lo menos que alguien se encargara de atender sus angustias cuando estas se volvían extremadamente amenazantes. El paciente necesita la seguridad de alguien quien pueda señalarle amable y firmemente qué es real y qué no, así como también apoyarlo y ayudarle a entender qué es lo que está atravesando. 
La percepción de algunos pacientes es que sus padres no pudieron cumplir este rol debido a que no fueron profesionales y no estuvieron lo suficientemente preparados para afrontar las experiencias de los pacientes en la manera adecuada, como por ejemplo decidir llevar al paciente donde profesionales de la salud. En contraste, algunos tienen la percepción de que sus padres empezaron a ayudarlos o apoyarlos únicamente a raíz de su enfermedad.

Los pacientes, en su mayoría, hicieron una evaluación negativa de sus vínculos familiares. No solo se producen escasos contactos entre ellos, sino que sienten que no les dan suficiente soporte y tienen la impresión que sus padres no tienen expectativas de ellos. Existe un sentimiento de marginalidad que va junto con deseos de más contactos. La marginalidad es sentida con mayor fuerza cuando los pacientes hablan de sus sentimientos de ser excluidos de la vida familiar. Esto de alguna manera es compensado por percepciones positivas en lo que se refiere a amigos, quienes en muchos casos reemplazan a los miembros familiares en el ámbito afectivo.

Varios pacientes hablaron espontáneamente de su estado de ánimo deprimido. Unos pacientes se refirieron a un ánimo triste, "bajo", mientras que otros hablaron de la falta de fuerzas o el agotamiento y la falta de ganas o voluntad para hacer las cosas. Cabe resaltar que son precisamente los pacientes con una mayor conciencia de enfermedad los que hablan de su estado de ánimo deprimido; quizá a ello se deba el que puedan describir su ánimo; sin embargo, podríamos pensar también que el darse cuenta de lo que les pasa los lleva a deprimirse. Muchos pacientes comentaron que este estado de ánimo los acompaña desde que enfermaron. Arieti (1974) señala haber encontrado en pacientes esquizofrénicos que tenían un ánimo deprimido una tendencia a una mala autoimagen con fuertes matices depresivos antes de que la psicosis empiece; sin embargo, luego de la instalación de la psicosis, los mecanismos proyectivos prevalecieron, lo que explicaría en parte el desarrollo de la esquizofrenia y no de algún trastorno específicamente afectivo. Cutting y Dunne (1989) encontraron 
que entre los primeros cambios asociados a la enfermedad, sus pacientes experimentaron cambios emocionales: ansiedad, miedo y depresión. El haber encontrado depresión en los pacientes entrevistados y el que esta empezara antes de los primeros síntomas psicóticos -como afirman Conrad (1964), Bustamante, Maurer y Loffler et al. (1994) y Addington et al. (1998) - nos hace pensar en ella como una parte constitutiva de la esquizofrenia, sea que la esquizofrenia comience con síntomas depresivos o sea que la depresión sea una consecuencia de las primeras manifestaciones de la esquizofrenia. Se trata de una vivencia que acompaña a los pacientes en la experiencia de la enfermedad. Por otro lado, el inicio temprano de una depresión podría ser un indicador de una esquizofrenia en potencia; esto nos hace pensar también en la depresión como una señal de alarma.

El miedo, referido también como pánico o terror, ha sido descrito por varios pacientes. Algunos contaron sobre el terror que tenían a todas las cosas desde pequeños. Coincidentemente, estos pacientes reportaron que desde antes de instalarse la enfermedad sentían que algo no andaba bien en ellos. Al igual que la depresión, el miedo parece ser un fenómeno constitutivo de la esquizofrenia, puesto que acompaña a los pacientes en todo su proceso. El miedo da indicios de que se está gestando algo, algo que se desconoce y no se sabe a dónde lo llevará a uno, lo que Jaspers (Conrad, 1964) describió como el "humor delirante": "me pasa algo, pero no sé qué [...]". Más adelante, el miedo se transforma en una serie de peligros que amenazan al paciente. Los peligros son conceptos-sentimientos que incluyen la inadecuación, el rechazo, la no-pertenencia, la culpabilidad, la marginalidad, los sentimientos de inferioridad, entre otros. Arieti (1974) afirma que debido a sus débiles defensas y a su sensibilidad por las experiencias pasadas, el paciente distorsiona el ambiente. "En este punto su distorsión no es aún una proyección paranoide ni una delusión en un sentido técnico, sino que es experimentada como angustia, vulnerabilidad, miedo, ansiedad y sufrimiento mental [...] todo parece inútil, sin sentido y aterrador", señala el autor. 
La intranquilidad fue mencionada por casi todos los pacientes en algún momento de la entrevista. Los que no la mencionan la tienen reportada en sus historias clínicas. Creemos que este es un común denominador de la enfermedad. Quienes la mencionan se dedican a describirla más que otras sensaciones, a veces con mucha intensidad, como una sensación que invade cuerpo y mente y no les permite pensar ni hacer otras cosas, sino preocuparse y no salir de un círculo vicioso de temores, falta de paz e inseguridad.

Las hipótesis psicoanalíticas en torno a las delusiones no se contradicen con otro tipo de hipótesis (como las neurobiológicas, las cuales se centran en los déficits cognitivos), sino que estas pueden complementarse (Kaliksztein, 2000). Para ambas corrientes de pensamiento, las falsas ideas del paciente siguen una estructura cognitiva, una lógica que está constituida de forma diferente que la de las personas normales. Arieti (1974) ha señalado que la nueva lógica le permitirá al paciente ver la realidad como él quiere o le va a proporcionar, por lo menos de manera parcial, una falsa realización de deseos y de esta manera su ansiedad disminuye. En los esquizofrénicos paranoides, por ejemplo, esta lógica peculiar es usada solo cuando ellos se enfrentan a sus conflictos y complejos personales, señala el autor. Van den Bosch (1995), por su lado, ha afirmado que las delusiones serían un intento de explicar las fallas cognitivas y por ello las considera manifestaciones secundarias del trastorno. "Serían resultado del desorden cognitivo primario, el cual interactúa con los intentos del paciente por comprender las experiencias extrañas", menciona el autor. "Los síntomas cognitivos y psicóticos son grandes consumidores de energía y es comprensible que los pacientes traten de protegerse a sí mismos", agrega el autor. La adopción de esta nueva manera de pensar es en todos los casos una manera de protegerse. Sea que los protejan de su insostenible mundo interno o de las vivencias que producen las fallas cognitivas, las delusiones parecen ser una estrategia que a expensas del sentido de realidad la distorsionan con el fin de compensar vivencias iniciales displacenteras y no aceptables por uno mismo, creando una realidad paralela. Racamier (1983) ha sido radical 
al señalar que "toda esquizofrenia es una defensa contra la catástrofe" (p. 61).

Se ha visto que la aparición de los déficits cognitivos de atención, aprendizaje, etc. suele ser temprana, por lo general en jóvenes en la etapa escolar. Esto podría ser un indicador del posterior desarrollo de la enfermedad, por lo que su detección podría tener un importante valor predictivo y una consecuente labor preventiva.

La abundancia y la naturaleza de los contenidos religiosos evocados por los pacientes esquizofrénicos reflejan la importancia que estos tienen en la vivencia de la enfermedad. Algunos autores han destacado que las delusiones y alucinaciones místicas experimentadas en los estados psicóticos comparten similitudes con las experiencias místicas de las personas en general. Happold (1975) ha señalado que durante la experiencia mística se trasciende al ego o se atraviesa un proceso de "pérdida del ego" temporal y que dicha experiencia le da un sentido de propósito al individuo y un sentido de integración. Esta experiencia unificadora y ego-trascendente sería "una manera de buscar la integración o de intentar sentirla, sea bajo el propio control o no", señala el autor. De acuerdo con Lovell (1997), en las etapas agudas de la esquizofrenia el sentido de uno mismo está alterado y sus límites son imprecisos; así, "la invasividad de experiencias cognitivas y perceptivas anómalas y el sentido extremo de apartamiento del mundo consensual perturba la dialéctica sujeto-objeto necesaria para la conciencia de uno mismo [...] Un self impreciso da pie a que se llene de 'ideas no reales', entendiendo el self como una capacidad indeterminada de engancharse o estar orientado en el mundo caracterizada por esfuerzo y reflexividad".

A diferencia de las otras delusiones, las delusiones místicas de los pacientes fueron por lo general placenteras, pudiendo tratarse justamente de un intento de buscar un sentido de unidad y de conexión con el mundo a otro nivel dado que en la psicosis se produce una disrupción del funcionamiento normal de la conciencia. Ante lo aterrorizante 
de las experiencias de daño, persecución, control, referencia, etc., las experiencias místicas brindarían al esquizofrénico otro tipo de vivencias afectivas, más armónicas y apuntando quizá a la reintegración de lo fragmentado, tratando de encontrar un sentido a lo que les está sucediendo.

Respecto de la religión, Corin (1990) ha señalado que la mayoría de pacientes tiene una especie de experiencia religiosa continua. No siempre se traduce en una práctica religiosa regular, su relación con Dios es más a menudo una cuestión de una orientación interna: pensar en Dios, hablar con él por horas, etc. Una paciente entrevistada mencionó incluso que esta fue la razón de una recaída. Para algunos pacientes el marco de referencia religioso puede estructurar toda su vida y ayudarlos así a protegerse y valorizar un espacio interno "a distancia" de la vida social. Las referencias religiosas ayudarían a dotar de significado a la vida y al universo y permitir a los pacientes situarse en el marco del mundo. Por otro lado, los grupos religiosos a los cuales se han adherido varios pacientes - promueven un sentido interno de pertenencia al grupo, inclusive si el paciente permanece de hecho muy aislado.

Respecto de los aspectos que contribuyen a una mejoría, resulta sorprendente que el $80 \%$ de los pacientes mencionó que el estar ocupados los ayudaría a sentirse mejor: trabajar, en la mayoría de casos, o estudiar en algunos otros. No sorprende, por el contrario, la frustración que deviene de los deseos de trabajar y no poder hacerlo. Al hablar de esto, los pacientes expresaban su impotencia de no poder "llevar una vida normal", "tener un trabajo que les dé ingresos" o "poder ser como los demás".

En vista de que no se encontró relación alguna entre el número de hospitalizaciones del paciente y el modo de experiencia subjetiva de la enfermedad, se podría pensar que la cantidad de hospitalizaciones no es lo que mantiene al paciente tranquilo en el largo plazo, ni lo que le brinda una mayor comprensión de su trastorno, sino la calidad de la 
atención que recibe el paciente durante las mismas. La experiencia subjetiva de la esquizofrenia tiene que ver también con un factor central que perpetúa las vivencias negativas de los pacientes: el ambiente al cual retorna el paciente al salir de alta. Y por lo general este no es alentador.

Los pacientes sienten que conversar los ayuda; ser escuchados y comprendidos, también. A veces mencionan que, a diferencia con el psiquiatra, el psicólogo dedica más tiempo y se interesa más por ellos. El psicólogo es visto también como un orientador frente a la falta de orientación por parte de los padres a la cual nos referimos anteriormente.

León (1992) ha señalado que los pacientes que lograron un modo de vivenciar la enfermedad, integrados por lo general, tienen una buena evolución clínica mientras que los que experimentan la enfermedad como falsificación tienen una mala evolución de la misma. Esto nos hace pensar en la posibilidad de orientar el tratamiento hacia el trabajo de ciertos aspectos que serían adecuados para cada tipo de paciente; quizá se podría agrupar en los hospitales a los pacientes en estos cuatro grupos con fines terapéuticos y tratarlos de manera más específica.

Podemos entender mejor a la persona si exploramos sus sentimientos a un nivel más directo, empático, en una conversación con ella sobre su propia experiencia de la esquizofrenia. Poner más énfasis en este tipo de acercamiento nos permite una mejor comprensión de lo que la persona está viviendo. Por otro lado, no debemos pasar por alto el rango de experiencias subjetivas de los pacientes ya que estas tienen una gran implicancia para la formulación de tratamientos más completos y consecuentemente más efectivos para los pacientes con esquizofrenia.

El miedo, la soledad, los sentimientos de extrañeza y de no pertenencia, la depresión y las frustraciones que provienen de las fallas 
cognitivas y de un mundo social insatisfactorio no siempre están expuestos a quienes interactúan con ellos. Por ello es necesario prestarles atención para que no queden camuflados con sintomatologías explícitas.

Creemos que replicar el presente estudio con una mayor cantidad y diversidad de pacientes enriquecería la investigación cualitativa de la esquizofrenia; ello tendría implicancias tanto para su comprensión como también para la terapéutica del trastorno.

\section{Referencias}

Addington, D., Addington, J. y Patten, S. (1998). Depression in people with first episode schizophrenia. British Journal of Psychiatry (Suppl. 33), 172, 90-92.

American Psychiatric Association (1994). Diagnostic and statistical manual of mental disorders (4ta. ed.). Washington, DC: APA.

Arieti, S. (1974). Interpretation of Schizophrenia (2da. ed.). Nueva York: Basic Books.

Atkinson, J. M. (1986). Esquizofrenia: una guía para los pacientes y sus familias. Buenos Aires: Planeta.

Bustamante, S., Maurer, K. y Loffler, W. (1994). Depression in the early course of schizophrenia. Fortschritte der NeurologiePsychiatrie, 62, 317-329.

Conrad, K. (1964). La esquizofrenia incipiente: Intento de un análisis de la forma del delirio. Madrid: Alhambra.

Corin, E. E. (1990). Facts and meaning in psychiatry. An anthropological approach to the lifeworld of schizophrenics. Culture, Medicine and Psychiatry, 14, 153-188.

Cutting, J. y Dunne, F. (1989). Subjective experience of schizophrenia. Schizophr. Bull., 15, 217-231.

Fromm-Reichmann, F. (1973). Psicoterapia intensiva en la esquizofrenia. Buenos Aires: Horme. 
Happold, F. C. (1975). Mysticism: A study and anthology. Harmondsworth: Penguin Books.

Kaliksztein, B. (2000). La experiencia subjetiva de la enfermedad en pacientes con esquizofrenia. Tesis de Licenciatura en psicología, Pontificia Universidad Católica del Perú, Lima.

León, C. A. (1992). El esquizofrénico y su trastorno: nociones subjetivas del devenir psicótico. Salud Mental, 15 (1), 24-34.

Lovell, A. (1997). The city is my mother: Narratives of schizophrenia and homelessness. American Anthropologist, 99 (2), 335-368.

Racamier, P. C. (1983). Los esquizofrénicos. Madrid: Biblioteca Nueva.

Randolph, C., Goldberg, T. E. y Weinberger, D. R. (1993). The neuropsychology of schizophrenia. En K. Y. Heilman y E. Valenstein (Eds.), Clinical Neuropsychology. Nueva York: Oxford University Press.

Roheim, G. (1959). Magia y esquizofrenia. Buenos Aires: Paidós.

Sechehaye, M.-A. (1958). La realización simbólica. Diario de una esquizofrénica. México: Fondo de Cultura Económica.

Van den Bosch, R. J. (1995). Schizophrenia: Inner and outer worlds. Communication and Cognition, 28, 327-342.

Yung, A. R., Phillips, L. J., McGorry, P. D., McFarlane, C. A., Francey, S., Harrigan, S., Patton, G. C. y Jackson, H. J. (1998). Prediction of psychosis: A step towards indicated prevention of schizophrenia. British Journal of Psychiatry (Suppl. 33), 172, 14-20. 